\title{
KIC 8462852 FADED THROUGHOUT THE KEPLER MISSION
}

\author{
Benjamin T. Montet ${ }^{1,2}$ and Joshua D. Simon ${ }^{3}$ \\ ${ }^{1}$ Cahill Center for Astronomy and Astrophysics, California Institute of Technology, Pasadena, CA, 91125, USA; btm@astro.caltech.edu \\ ${ }^{2}$ Harvard-Smithsonian Center for Astrophysics, Cambridge, MA 02138, USA \\ ${ }^{3}$ Observatories of the Carnegie Institution of Washington, 813 Santa Barbara St., Pasadena, CA 91101, USA \\ Received 2016 August 3; revised 2016 September 30; accepted 2016 September 30; published 2016 October 20
}

\begin{abstract}
KIC 8462852 is a superficially ordinary main sequence F star for which Kepler detected an unusual series of brief dimming events. We obtain accurate relative photometry of KIC 8462852 from the Kepler full-frame images, finding that the brightness of KIC 8462852 monotonically decreased over the four years it was observed by Kepler. Over the first $\sim 1000$ days KIC 8462852 faded approximately linearly at a rate of $0.341 \pm 0.041 \% \mathrm{yr}^{-1}$, for a total decline of $0.9 \%$. KIC 8462852 then dimmed much more rapidly in the next 200 days, with its flux dropping by more than $2 \%$. For the final $\sim 200$ days of Kepler photometry the magnitude remained approximately constant, although the data are also consistent with the decline rate measured for the first 2.7 years. Of a sample of 193 nearby comparison stars and 355 stars with similar stellar parameters, none exhibit the rapid decline by $>2 \%$ or the cumulative fading by $3 \%$ of KIC 8462852 . Moreover, of these comparison stars, only one changes brightness as quickly as the $0.341 \% \mathrm{yr}^{-1}$ measured for KIC 8462852 during the first three years of the Kepler mission. We examine whether the rapid decline could be caused by a cloud of transiting circumstellar material, finding that while such a cloud could evade detection in submillimeter observations, the transit ingress and duration cannot be explained by a simple cloud model. Moreover, this model cannot account for the observed longer-term dimming. No known or proposed stellar phenomena can fully explain all aspects of the observed light curve.
\end{abstract}

Key words: circumstellar matter - methods: data analysis - stars: individual (KIC 8462852) - stars: variables: general - techniques: photometric

Supporting material: data behind figures

\section{INTRODUCTION}

In addition to its primary mission of detecting exoplanets (Borucki et al. 2010), the Kepler satellite's exquisite photometry has allowed groundbreaking studies in stellar astrophysics. Most notably, analyses of the seismic modes of stellar light curves have enabled otherwise inaccessible measurements of stellar ages, masses, evolutionary states, and internal structure (e.g., Bedding et al. 2011; Mosser et al. 2012; Bastien et al. 2013; Chaplin et al. 2014; Silva Aguirre et al. 2015). Kepler data are also providing fundamental new insights into mass-loss on the red giant branch (e.g., Miglio et al. 2012), the variation of stellar rotation and stellar dynamos with age (e.g., Meibom et al. 2015; Barnes et al. 2016; van Saders et al. 2016), and the origin of the Blazhko effect in RR Lyrae variables (e.g., Szabó et al. 2010).

One of the most confounding Kepler discoveries is the light curve of KIC 8462852. First presented by Boyajian et al. (2016), KIC 8462852 appears to be a typical F3V star (Lisse et al. 2015). However, its light curve exhibits 10 significant dips over the time-span of the Kepler mission. These dips are irregular in shape, aperiodic, and vary in depth from fractions of a percent up to $20 \%$ of the total flux of the star. While the dips as a whole do not obey an obvious periodicity, a subset of them are consistent with a period of $\sim 48$ days, although some of those are $180^{\circ}$ out of phase with the others. Boyajian et al. present several physical models to account for these dipping events, concluding that a large family of exocomets or planetesimal fragments orbiting the star could plausibly explain the data.

KIC 8462852 quickly became the focus of considerable attention. Thompson et al. (2016) place tight constraints on the circumstellar dust around KIC 8462852 from millimeter and submillimeter observations, ruling out the planetesimal fragment hypothesis; Marengo et al. (2015) reach the same conclusion from an analysis of warm Spitzer data. Bodman \& Quillen (2016) model the light curve with a swarm of comets. They find that the KIC 8462852 light curve in Quarters 16 and 17 of the Kepler mission can be explained by approximately 100 comets in a very tight cluster. This model, however, is unable to match the earlier dips in the light curve. Wright et al. (2016) proposed that the star may make an ideal target for SETI programs. So far, SETI searches in the optical (Abeysekara et al. 2016; Schuetz et al. 2016) and radio (Harp et al. 2016) have only resulted in null detections.

Other studies have made use of archival observations of KIC 8462852. Schaefer (2016) analyzed photographic plates from the "Digital Access to a Sky Century @ Harvard" (DASCH) archive (Grindlay et al. 2009; Laycock et al. 2010), obtained between 1890 and 1989. This work found KIC 8462852 to be dimming at an average rate of $0.165 \pm 0.013$ mag century $^{-1}$, or $0.152 \pm 0.012 \% \mathrm{yr}^{-1}$. This result was quickly called into question. Both Hippke et al. (2016) and Lund et al. (2016) suggest that the observed dimming is the result of systematics in the DASCH data, particularly the Menzel Gap of the 1950s, and report the flux of KIC 8462852 to be consistent with no change over the baseline of the DASCH plates. However, the DASCH team has not found evidence for such systematics (Laycock et al. 2010; Tang et al. 2013a, 2013b). Additionally, the claimed systematics appear to depend on the choice of reference stars (J. Grindlay 2016, private communication).

If the star is dimming with time, then a survey with a long time baseline and high photometric precision would be able to detect this variation. Modern ground-based imaging surveys generally operate over timescales of $\sim 5$ years, and they lack the 
photometric accuracy to detect the $\sim 8$ mmag variation that would be expected from the analysis of the DASCH data by Schaefer (2016). The best-calibrated existing surveys, SDSS and Pan-STARRS (Magnier et al. 2013; Finkbeiner et al. 2016), would need a time baseline $\sim 5$ times as long in order to measure such a brightness change at $5 \sigma$ significance (ignoring that stars as bright as KIC 8462852 with $V=11.7$ are badly saturated in such data sets). Data from the Kepler mission, however, provide exactly such an opportunity.

The Kepler mission was designed to detect and characterize transit events with timescales of minutes to hours. To that end, Kepler processing pipelines aim to remove longer-term trends in the data caused by instrumental effects. This process is welldescribed by Jenkins et al. (2010) and García et al. (2011) and is the basis for the creation of the "pre-search data conditioning" (PDC) light curves. The PDC light curves, among many other corrections, remove long-term trends in the observed flux of stars that correlate with either trends from nearby stars or with the centroid of a star's motion during an observing quarter. The main source of target motion is differential velocity aberration, which leads to an approximately linear drift in the centroid of a star at the 0.1 pixel level over the course of a quarter. Any signals that are approximately linear over a quarter will then be removed as an instrumental artifact. Therefore, while the Boyajian et al. (2016) analysis of KIC 8462852 was sensitive to short-timescale variability, they or any other group analyzing long-cadence photometry of the target will not be able to detect any long-term trends. The end result is that signals with characteristic timescales longer than a single observing quarter will not appear in the long-cadence data. Searches for rotation periods in Kepler data corroborate this claim. McQuillan et al. (2014) identified more than 34,000 rotation periods for stars in the Kepler field, but their detection efficiency drops for periods above 40 days and they do not detect any rotation periods longer than 70 days. Other sets of light curves designed to detect transiting planets will more aggressively remove stellar variability through a "de-trending" process, removing all non-transit signals (e.g., Carter et al. 2012).

The pixel-level data delivered by the telescope present a better opportunity to preserve long-term trends in the Kepler light curves. Photometry with the pixel-level data has its own difficulties. Aperture photometry is complicated, as the apertures around each star recorded by the telescope and downlinked to Earth are often smaller than the full point-spread function (PSF) of the star (Bryson et al. 2010). Small deviations in the pointing of the telescope can then cause large variations in the total flux recorded inside the photometric aperture as the edges of the star's PSF move relative to the edge of the downloaded set of pixels. Instead, one could consider modeling the PSF of the star on the detector. While possible in some cases (e.g., Rappaport et al. 2014), the lack of a reliable flatfield for the Kepler detector array and the lack of background "sky" pixels make PSF modeling difficult, and nearly impossible for saturated stars $\left(K_{p}<12\right.$, Szabó et al. 2010).

An alternative approach to search for long-term linear trends is to leverage the full-frame images (FFIs) collected during the Kepler mission. At the beginning of the mission, Kepler recorded a series of eight FFIs over two days. It then continued to record one FFI each month throughout the mission (Haas et al. 2010). As Kepler rotated by $90^{\circ}$ every three months, there were generally three consecutive FFIs, with all stars located on the same pixels. A star then cycled through four different detectors over the course of a year, returning to its original position and repeating the cycle a year later. Because there are $\sim 13$ observations with any given star landing on the same pixels, accurate relative photometry can be obtained without flat-fielding. The FFIs have been used to study stellar variability in RR Lyrae stars in the Kepler field (Kinemuchi 2011), highlighting their utility for photometry.

The primary Kepler mission lasted four years. A star dimming at a linear rate of 0.165 mag century $^{-1}$ $\left(0.152 \pm 0.012 \% \mathrm{yr}^{-1}\right)$, as has been purported for KIC 8462852 (Schaefer 2016), would be expected to decrease in brightness by $0.6 \%$ over the Kepler baseline, well above the photometric precision of the telescope. By analyzing the FFI data, one could avoid the removal of this trend by the data processing pipeline and the loss of flux from limited apertures in the raw pixel-level data. In this study, we attempt to characterize the long-term photometric behavior of KIC 8462852 through an analysis of Kepler FFI data.

This paper is organized as follows. In Section 2, we describe our data analysis and photometry. In Section 3, we present our results. In Section 4, we compare our results to a similar analysis of stars close to KIC 8462852 on the detector and stars with similar stellar properties. In Section 5, we rule out instrumental effects that could be responsible for apparent brightness changes and consider whether background contamination, a transiting cloud of material, or a polar spot can explain the observed FFI light curve. We present our conclusions in Section 6.

\section{DATA ANALYSIS}

We downloaded all 53 FFI images from the Mikulski Archive for Space Telescopes (MAST) and sorted by the season in which they were collected. In this work, we use the "cal" frames, which have been calibrated from the raw data (Caldwell et al. 2010; Jenkins et al. 2010; Quintana et al. 2010). Specifically, these frames have had a bias level and dark current be subtracted, the smear from CCDs being illuminated during readout be corrected, the gain be divided, and a flat-field correction be applied. We removed one frame, 2009170043915, as it was collected with the spacecraft mispointed by four pixels on the sky, making calibration with the other frames in that spacecraft orientation unfeasible. Fluxes from the 2009170043915 frame differ from the other frames in that particular orientation taken during that same quarter by $0.5 \%$, suggesting that the offset in the flat-field from pixel to pixel is at the sub-percent level. For the remaining 52 frames, we first select a $120 \times 120$ pixel $(8$ arcmin square) region of the detector centered on the target star (Figure 1). As a part of the FFI data processing, a background has already been subtracted. Unlike in the $K 2$ mission, which observes in the ecliptic and detects a changing background due to increasing zodiacal light during each campaign (Molnár et al. 2015), the background level is low and does not significantly change during the Kepler mission.

We begin our processing by masking any saturated stars. While Kepler was designed with preservation of photometry of saturated stars in mind, recovering the absolute brightness of these stars requires careful placement of photometric apertures, making automated procedures challenging. We then identify 


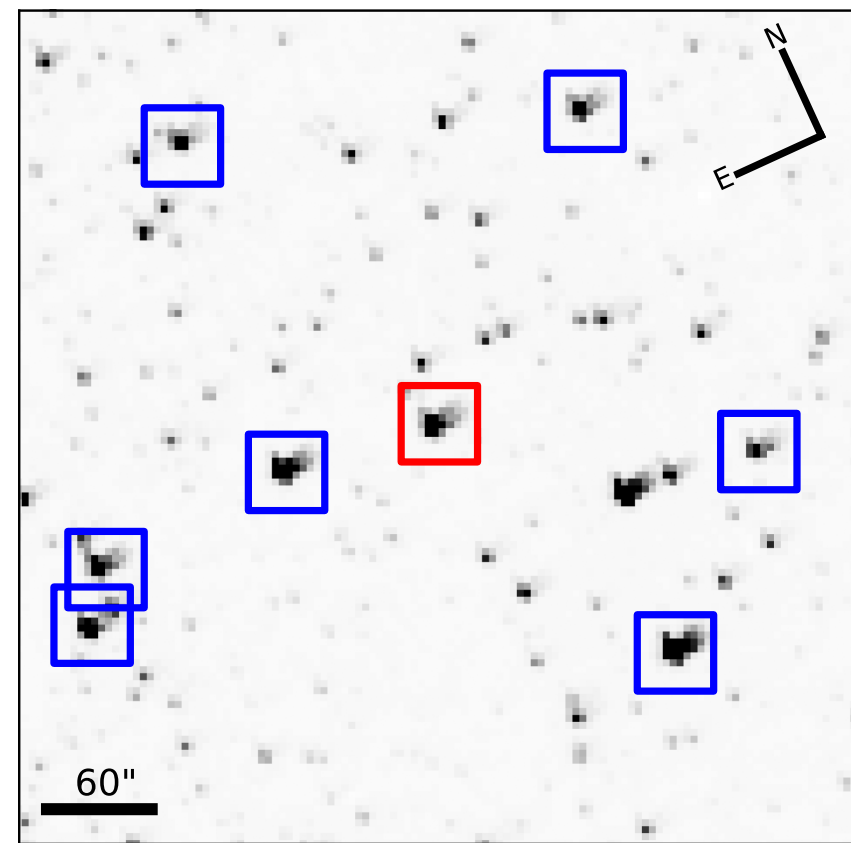

Figure 1. "Postcard" region of the Kepler detector surrounding KIC 8462852, located at the center of the image. In red is the aperture used for measuring the photometry from this star in all frames. The seven blue apertures represent the reference stars used for comparison photometry. The frame is aligned to match the orientation of the telescope, with north rotated approximately $25^{\circ}$ from pointing up. Most of the stars visible in this image were not observed in long or short cadence during the Kepler mission; the FFI data are the only photometry available for these targets.

the 10 brightest nearby stars in the subframes, based on their brightest central pixel.

For all selected stars, including our target, we perform aperture photometry. The Kepler telescope is defocused to produce an image with a full-width at half-maximum of 1.5 pixels in diameter. However, due to the location of KIC 8462852 in the corner of the Kepler field, the Schmidt optics of the telescope produce a non-Gaussian and asymmetric PSF for each star (Bryson et al. 2010). As can be seen in Figure 2, these PSFs are several pixels in size and extend well beyond the pixels downloaded at a 30-minute cadence during standard Kepler observations. To collect all the flux from the star, we then create $11 \times 11$ pixel apertures centered on the brightest pixel of each star.

Not all of these stars may be appropriate as calibration stars for relative photometry; some may be intrinsically variable, while others may be too faint to reliably obtain accurate photometry in each single exposure. We discard all stars with a standard deviation of the residuals from a linear fit to the data larger than $0.5 \%$. Such a cut removed one bright star near KIC 8462852 (KIC 8462738) with periodic variations at the $>5 \%$ level, as well as the two faintest stars in the sample, where photon noise prohibited accurate relative photometry, leaving us with seven reference stars.

We then determine the flux level of KIC 8462852 by comparing its aperture photometry to the aperture photometry recorded from the calibrator stars, weighted by the signal-tonoise ratios of each of the calibration stars. We use the standard deviation of the flux measured among all observations recorded on that particular pixel as the uncertainty associated with each observation. The scatter between points on a particular detector can vary by as much as a factor of two from detector to

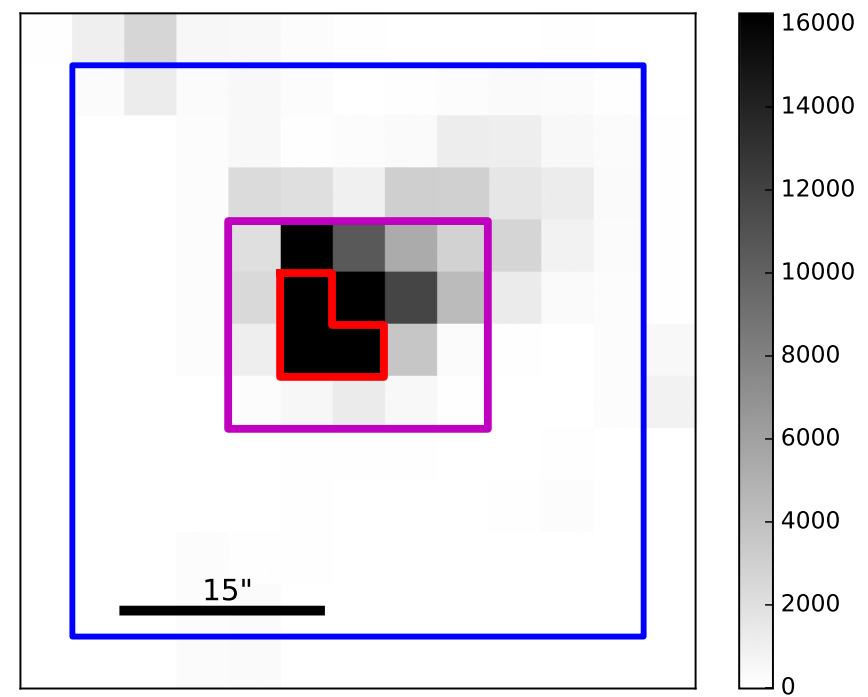

Figure 2. Zoomed region of Figure 1 centered on KIC 8462852. In blue is the aperture used for measuring the photometry from this star in all frames. In purple is the postage stamp downloaded for the creation of the long-cadence light curve for this star, while in red are the actual pixels used in the creation of the SAP and PDC light curves in Quarter 0 of the mission. The pixel response function is wider than the regions downloaded at a 30-minute cadence, so not all of the flux from KIC 8462852 is captured. The orientation here is the same as in Figure 1.

detector, in line with previous analyses of the noise in the primary Kepler mission (Gilliland et al. 2011).

Finally, we combine the data from each separate channel on the telescope. Because of the uncertainty in the underlying flatfield, we expect an offset between each telescope orientation, which is not known a priori, qualitatively similar to those observed in the Kepler long-cadence data between quarters. We first normalize the flux values as recorded in the previous paragraph by dividing the observations from each particular channel by their mean value. We then apply a linear offset term between each channel, so that if the sensitivity of one particular channel is lower than the others, our model would not try to model that signal's actual astrophysical variation. In practice, this requires fitting three parameters that define the offset for each channel relative to the first channel. The results are shown in Figure 3 and Table 1. For display purposes, in Figure 3 we must select a particular set of offsets. We choose the maximum likelihood model of a linear fit to the first three years of data from the Kepler mission, which minimizes the scatter from quarter to quarter. In our analysis of any long-term dimming, we allow the offset terms to float as free parameters.

\section{RESULTS}

\subsection{A Long-term Dimming}

From Figure 3, three main features are apparent. The first is a long-term dimming at a linear rate from the beginning of the Kepler mission until approximately Quarter 13. The dimming then increases rapidly such that the observed flux from the star decreases by approximately $2.5 \%$ over 200 days. For the final 200 days of the Kepler mission, the light curve flattens out, either returning to the original rate of decay or remaining constant.

In the primary Kepler mission, data were collected in four orientations as the telescope rolled every 93 days to keep its heat shield pointed at the Sun. As a result, data for each star are 

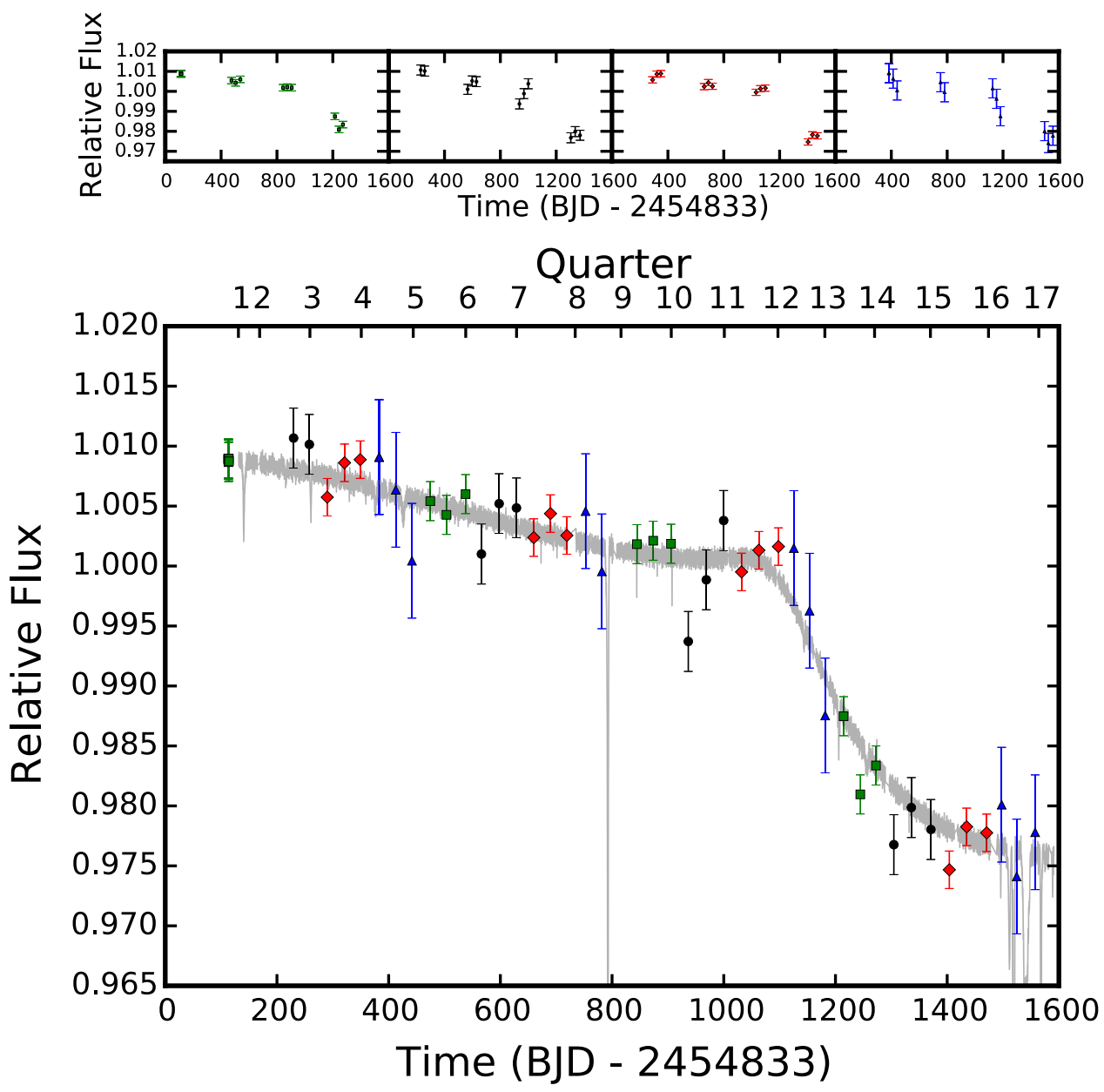

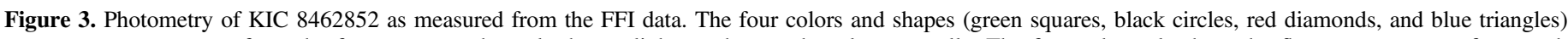

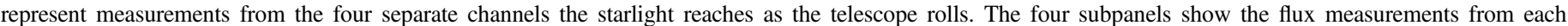

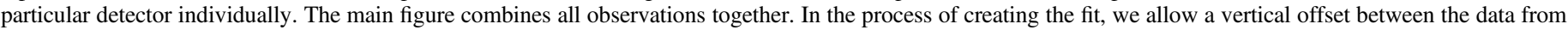

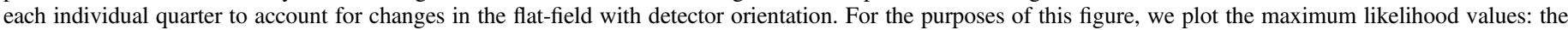

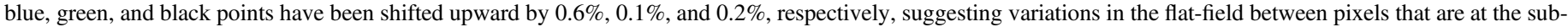

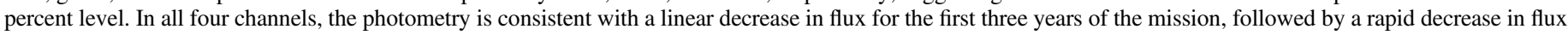

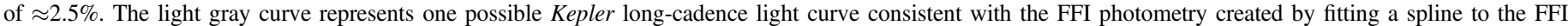
photometry as described in Section 4. The large dips observed by Boyajian et al. (2016) are visible but narrow relative to the cadence of FFI observations.

(The data used to create this figure are available.)

collected on four different pixels, each for three months of every year. Therefore, we can separate many instrumental effects caused by a faulty pixel on the detector from astrophysical phenomena by looking for the same trends when only considering data from each specific orientation. In practice, we allow for a linear offset between the observed fluxes recorded on each individual module, reflecting our uncertainty about the underlying flat-field of the detector itself. We note that these results are apparent in data from each individual detector, not just the combined light curve, suggesting that the decline in flux is an astrophysical effect rather than an instrumental one, as discussed more fully in Section 5 .

We fit a line to the first region of data, from the beginning of the mission until Barycentric Julian Date (BJD) 2456003 (or BJD-2454833 = 1170), encompassing 39 data points. We allow the relative offset between each detector to vary, three in total to account for possible differences in the flat-field between different detectors. It is possible that the recorded measurement uncertainties are not representative of the actual uncertainties.
To account for a possible systematic underestimation of the uncertainties, we allow for an extra uncertainty term applied to data from each detector to be added in quadrature to our recorded uncertainties estimated from the data themselves, so that the $i$ th data point recorded in the $q$ th telescope orientation has uncertainty $s_{i, q}$ such that

$$
s_{i, q}=\sqrt{\sigma_{i}^{2}+j_{q}^{2}}
$$

Here, $\sigma_{i}$ is the uncertainty for the $i$ th observation (as described in Section 2) and $j_{q}$ is the level of underestimation of the photometric uncertainty in quarter $q$, similar to the concept of jitter in radial velocity (RV) observations (Butler et al. 1996). We assume that all observations on a given detector have the same $j_{q}$ value, so that there are four $j_{q}$ values fit in our analysis.

Including a slope and zeropoint for the linear fit, there are a total of nine free parameters: the slope, zeropoint, three offset terms describing the linear offset for each quarter relative to the first, and four "jitter" terms describing the excess photometric 
Table 1

FFI Data

\begin{tabular}{|c|c|c|c|c|c|c|}
\hline $\begin{array}{l}\text { Time } \\
\text { (BJD-2454833) }\end{array}$ & $\begin{array}{l}\text { Observed Flux } \\
\quad\left(\mathrm{e} \mathrm{s}^{-1}\right)\end{array}$ & $\begin{array}{l}\text { Reference Flux }{ }^{\mathrm{a}} \\
\quad\left(\mathrm{e} \mathrm{s}^{-1}\right)\end{array}$ & Flux Ratio & Normalized Flux ${ }^{\mathrm{b}}$ & $\begin{array}{l}\text { Uncertainty } \\
\qquad(1 \sigma)\end{array}$ & Orientation \\
\hline 112.742 & 277998 & 1384241 & 0.200831 & 1.00896 & 0.00163 & 3 \\
\hline 112.867 & 278052 & 1384642 & 0.200811 & 1.00886 & 0.00157 & 3 \\
\hline 113.018 & 278029 & 1384472 & 0.200820 & 1.00890 & 0.00158 & 3 \\
\hline 113.234 & 277993 & 1384621 & 0.200772 & 1.00867 & 0.00161 & 3 \\
\hline 113.338 & 278018 & 1384317 & 0.200834 & 1.00898 & 0.00161 & 3 \\
\hline 113.550 & 278053 & 1384618 & 0.200816 & 1.00888 & 0.00163 & 3 \\
\hline 113.733 & 278046 & 1384486 & 0.200830 & 1.00895 & 0.00162 & 3 \\
\hline 114.166 & 277968 & 1384404 & 0.200785 & 1.00873 & 0.00165 & 3 \\
\hline 229.825 & 283733 & 1408715 & 0.201413 & 1.01067 & 0.00257 & 0 \\
\hline 258.006 & 283423 & 1407913 & 0.201307 & 1.01014 & 0.00267 & 0 \\
\hline 290.086 & 268042 & 1332411 & 0.201171 & 1.00574 & 0.00153 & 1 \\
\hline 320.980 & 268832 & 1332528 & 0.201746 & 1.00861 & 0.00162 & 1 \\
\hline 349.037 & 268720 & 1331627 & 0.201798 & 1.00887 & 0.00156 & 1 \\
\hline 382.955 & 275452 & 1377897 & 0.199908 & 1.00908 & 0.00484 & 2 \\
\hline 383.035 & 275495 & 1378097 & 0.199910 & 1.00909 & 0.00483 & 2 \\
\hline 412.766 & 275353 & 1381124 & 0.199369 & 1.00636 & 0.00460 & 2 \\
\hline 441.740 & 273902 & 1381975 & 0.198196 & 1.00044 & 0.00502 & 2 \\
\hline 474.535 & 276125 & 1379789 & 0.200121 & 1.00541 & 0.00156 & 3 \\
\hline 503.428 & 275547 & 1378483 & 0.199891 & 1.00427 & 0.00154 & 3 \\
\hline 537.695 & 275378 & 1375253 & 0.200238 & 1.00600 & 0.00173 & 3 \\
\hline 566.057 & 279781 & 1402980 & 0.199419 & 1.00100 & 0.00256 & 0 \\
\hline 597.811 & 280641 & 1400955 & 0.200321 & 1.00520 & 0.00248 & 0 \\
\hline 628.829 & 280421 & 1400350 & 0.200251 & 1.00485 & 0.00266 & 0 \\
\hline 659.806 & 265617 & 1324798 & 0.200496 & 1.00238 & 0.00153 & 1 \\
\hline 689.762 & 266120 & 1324664 & 0.200896 & 1.00437 & 0.00157 & 1 \\
\hline 719.084 & 265682 & 1324891 & 0.200531 & 1.00255 & 0.00158 & 1 \\
\hline 752.576 & 272275 & 1368110 & 0.199015 & 1.00458 & 0.00483 & 2 \\
\hline 781.739 & 271728 & 1372220 & 0.198021 & 0.99956 & 0.00462 & 2 \\
\hline 844.444 & 273245 & 1370321 & 0.199402 & 1.00183 & 0.00166 & 3 \\
\hline 873.644 & 272944 & 1368418 & 0.199922 & 1.00211 & 0.00163 & 3 \\
\hline 905.459 & 272206 & 1365061 & 0.199409 & 1.00186 & 0.00159 & 3 \\
\hline 936.477 & 275546 & 1391437 & 0.198030 & 0.99371 & 0.00247 & 0 \\
\hline 968.762 & 276586 & 1389492 & 0.199055 & 0.99885 & 0.00236 & 0 \\
\hline 999.801 & 277627 & 1387847 & 0.200415 & 1.00380 & 0.00248 & 0 \\
\hline 1031.800 & 263255 & 1316791 & 0.199922 & 0.99951 & 0.00153 & 1 \\
\hline 1062.757 & 263785 & 1317059 & 0.200283 & 1.00132 & 0.00151 & 1 \\
\hline 1097.862 & 263738 & 1316417 & 0.200345 & 1.00162 & 0.00156 & 1 \\
\hline 1125.427 & 269393 & 1357792 & 0.198405 & 1.00150 & 0.00453 & 2 \\
\hline 1153.523 & 268327 & 1359507 & 0.197371 & 0.99628 & 0.00474 & 2 \\
\hline 1181.558 & 266378 & 1361569 & 0.195641 & 0.98754 & 0.00505 & 2 \\
\hline 1214.517 & 266884 & 1357982 & 0.196530 & 0.98748 & 0.00168 & 3 \\
\hline 1244.453 & 264824 & 1356515 & 0.195224 & 0.98096 & 0.00166 & 3 \\
\hline 1272.590 & 264792 & 1353002 & 0.195707 & 0.98337 & 0.00166 & 3 \\
\hline 1304.527 & 268808 & 1380987 & 0.194649 & 0.97677 & 0.00250 & 0 \\
\hline 1335.832 & 269135 & 1378297 & 0.195266 & 0.97986 & 0.00237 & 0 \\
\hline 1370.855 & 268435 & 1377283 & 0.194902 & 0.97803 & 0.00256 & 0 \\
\hline 1403.835 & 255025 & 1308199 & 0.194944 & 0.97467 & 0.00168 & 1 \\
\hline 1434.914 & 255839 & 1307564 & 0.195661 & 0.97825 & 0.00150 & 1 \\
\hline 1470.673 & 255686 & 1307448 & 0.195561 & 0.97775 & 0.00156 & 1 \\
\hline 1497.564 & 261275 & 1345623 & 0.194167 & 0.98010 & 0.00469 & 2 \\
\hline 1524.495 & 260101 & 1347812 & 0.192980 & 0.97411 & 0.00456 & 2 \\
\hline 1557.495 & 261323 & 1349036 & 0.193711 & 0.97780 & 0.00496 & 2 \\
\hline
\end{tabular}

Notes.

"The "Reference Flux" is the sum of the fluxes of the seven reference stars, weighted by the signal-to-noise ratio of each star on the detector.

b The "Normalized Flux" is the Flux Ratio for each star normalized by quarter, as described in Section 2.

noise in each channel above the listed uncertainties. The resultant fit estimates the excess noise required in order to explain the data for the first three years of the mission as a straight line, and estimates the offset in flux between the different spacecraft orientations under the assumption that the rate of change of flux is constant over the first three years of the mission. We note that the data shown in Figure 3 and Table 1 are the uncertainties from the data themselves $\left(\sigma_{i}\right)$ rather than $s_{i, q}$.

By marginalizing over all parameters except the slope, we can measure the decay rate of the observed flux. We determine that the star is fading from our perspective at a rate of 
$0.341 \pm 0.041 \% \mathrm{yr}^{-1}$, or $3.41 \pm 0.41$ parts per thousand (ppt) $\mathrm{yr}^{-1}$. This is equivalent to fading at a rate of $0.370 \pm 0.044 \mathrm{mag}$ per century, exceeding the purported 0.165 mag century $^{-1}\left(0.152 \pm 0.012 \% \mathrm{yr}^{-1}\right)$ rate of dimming over the duration of the DASCH plates (Schaefer 2016) by more than a factor of two. The total change in brightness over this portion of the Kepler light curve is almost 1\%. The four maximum likelihood "jitter" values are $2.8,0.52,1.1$, and 0.28 parts per thousand, corresponding to orientations $0,1,2$, and 3 , respectively, in the parlance of Table 1, suggesting that the recorded uncertainties listed there are not significantly underestimated.

\subsection{A Rapid Dimming}

Beginning around or BJD-2454833 1100, lasting for approximately 200 days, the rate of dimming increases dramatically. Repeating the above exercise in the region between dates 1100 and 1250, we measure a decay rate of $3.37 \pm 1.48 \% \mathrm{yr}^{-1}$. Here, we allow the break points at which the slope changes to float as free parameters, and the uncertainty in the actual dimming rate during this event is dominated by the uncertainty in the exact timing of the change in slope. Regardless of the exact rate at which the star appears to dim from our line of sight, it is clear from the Kepler data that the star becomes approximately $2.5 \%$ dimmer over a period of 200 days. This rapid dimming is apparent in data from all four orientations of the Kepler spacecraft. We note that we would expect the Kepler PDC pipeline to remove signals such as this one in the pre-processing of the data, explaining why this signal does not appear in the originally published long-cadence light curve.

After the conclusion of this event, there are only nine epochs of FFI data. Over this range, the rate of change of flux from the star is poorly measured: it is consistent with the original rate of dimming, but also consistent with a flat light curve until the final FFI image is collected at the end of Quarter 16 of the Kepler mission.

We offer no definitive explanation that could explain the observed light curve in this work. The effect could be stellar in nature, although there are no known mechanisms that would cause a main sequence $\mathrm{F}$ star to dim in brightness by $2.5 \%$ over a few months. The effect could also be caused by a passing dust cloud in orbit around KIC 8462852. Indeed, the light curve at times larger than 1000 days has a morphology broadly similar to a transit event, although on markedly different timescales. We discuss this morphology more fully in Section 5.5.

\subsection{Comparison with Dimming Events}

The long-cadence light curve as observed by Kepler contains features in which the observed flux from the star decreases by as much as $20 \%$ for a few days at a time. Our results could be substantially affected if any of these events were to overlap with the collection of a full-frame image. We can test this possibility by comparing the times recorded for the FFI data with the largest dipping events in the Kepler light curve. The result is shown in Figure 4.

We find that the dips do not overlap with any of the FFI observations. The only FFI image that is sufficiently close to a dipping event to possibly be affected is the final FFI of Quarter 8. If we repeat our analysis and remove this data point, we recover the same long-term dimming; none of the results in this

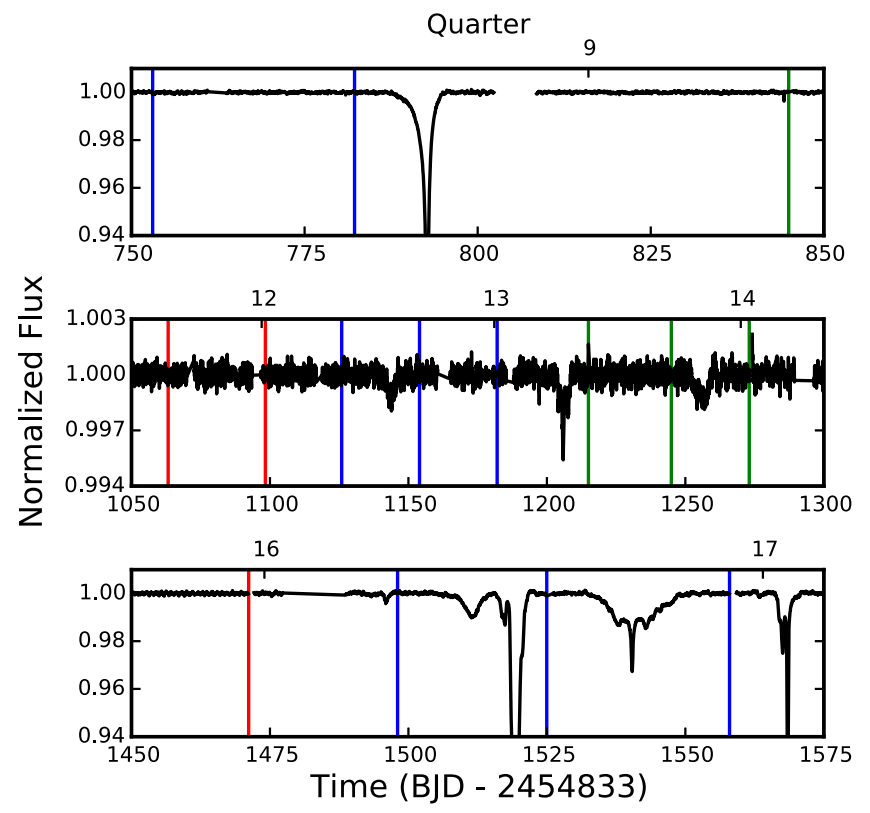

Figure 4. Times of FFI data collection superimposed on the Kepler longcadence light curve of KIC 8462852. Each vertical line represents the time of capture of an FFI, with the colors retaining their same meaning as in Figure 3. None of the FFIs are taken during a dip that could significantly affect our photometry. We produce our own long-cadence light curve as explained in Section 5.3, finding no evidence for the rise before the dip at the end of Quarter 8 observed in the PDC light curve.

(The data used to create this figure are available.)

work are significantly affected by the inclusion or exclusion of this single data point.

FFI images are typically collected as the last observation of a month of continuous data collection immediately before data downlink to Earth. After the FFI is taken, there is typically a $\approx 1$ day gap before data collection is resumed. It is unlikely that a large dip could fall in one of these gaps while evading detection in the long-cadence data, as the ingress duration for a dip would need to be smaller than the 30 minutes between the last long-cadence image of a single month and the FFI collected immediately afterward.

\subsection{Comparison with Rising Events}

As can be seen in Figure 1(b) of Boyajian et al. (2016), some of the dipping events are surrounded by apparent flux increases of $\sim 0.1 \%$. If these increases are real, they could be the signature of forward-scattering from dust grains (e.g., Rappaport et al. 2012). Most notably, the PDC light curve produced by the Kepler mission appears to rise just before a $15 \%$ dip. This particular FFI frame records a lower flux value than its predecessor by approximately $1 \mathrm{mmag}$, although the two are consistent with no change in flux. To test whether the small rise in the PDC light curve is induced by the initial data processing in the creation of the PDC light curves or if it is real, we create our own long-cadence light curve from the pixel-level data.

We perform aperture photometry using the entire postage stamp downloaded by the Kepler mission, not just the smaller aperture selected by the Kepler team for the creation of SAP and PDC light curves. We do the same for KIC 8462934, the single star within 2 arcmin with a $K_{p}$ within one magnitude of KIC 8462852. We then divide the flux recorded for KIC 
8462852 by the flux recorded for its apparent neighbor to account for instrumental trends.

In the full light curves, we detect a significant variation in the total flux received from quarter to quarter, a sign of changes in the underlying flat-field from channel to channel. For each quarter, we fit an offset term to minimize the variation between quarters (see Section 3.1). All light curves produced from Kepler data contain long-term variability that can result from a combination of astrophysical and instrumental effects. With the FFI data, we are able to use the nearby stars on the detector to separate the astrophysical effects from any instrumental effects shared by nearby stars. However, as the vast majority of stars in the Kepler Input Catalog were not targeted for regular photometry during the Kepler mission, there is a paucity of stars with long-cadence data to compare against to robustly detect long-term astrophysical trends in the long-cadence data. In this case, for the purposes of plotting Figure 4 we remove long-term trends. We divide the light curve into distinct regions separated by gaps in the data of at least 0.5 days. We then mask the regions of the light curve identified as short-term dips by Boyajian et al. (2012) and fit a spline to the remainder of the data, dividing the measured light curve by the spline to flatten the light curve. The result is a normalized light curve that removes trends but preserves the shape and magnitude of the rapid dips. These data are shown in Figure 4 and we make them publicly available as "data behind the figure" associated with Figure 3.

Once we have re-processed the light curve, we can compare the rising events to those observed in the PDC light curve. The rise in the PDC light curve in Q8 does not exist in our reprocessed light curve, suggesting that it is an artifact of the processing in the development of the PDC light curve. Similarly, the rise in the light curve at Day 1148, observed in the PDC light curve and this light curve, is almost certainly an artifact of data processing. There are also small rises observed before the dips at dates 140 and 260 . We note that these also occur immediately after data downlinks, as the telescope is reestablishing thermal balance after rotating to point to Earth and changing the position of its heat shields. It is well established that this maneuver leads to a ramp in the observed flux that lasts $\approx 3$ days after observations resume (Smith et al. 2012). As both rises occur during these windows, it is likely that they are induced by these thermal effects. There is a small rise $(\approx 2 \mathrm{mmag})$ observed at the end of Quarter 13 that may be real; it appears at low significance in the FFI data as well.

While our re-processed light curve is useful for studying the short-term dimming and potential rising events in the light curve of Boyajian et al. (2016), it and other versions of the Kepler long-cadence data for this star are not well-suited for the detection of long-term variations in the observed flux (see Section 1). The total aperture recorded by Kepler at a 30minute cadence is smaller than the PSF of the detector, so small changes in the position of the star cause flux variations that easily exceed the magnitude of the long-term trend. Moreover, many of the nearby reference stars used in this work that should share systematics with KIC 8462852 were not observed at a 30 minute cadence, meaning the opportunity to co-trend systematics as robustly as done here is lost. The best opportunity to create a light curve that is accurate both on traditional Kepler timescales and over years is to combine the FFI data with longcadence data, as we discuss in Section 5.3. This light curve can

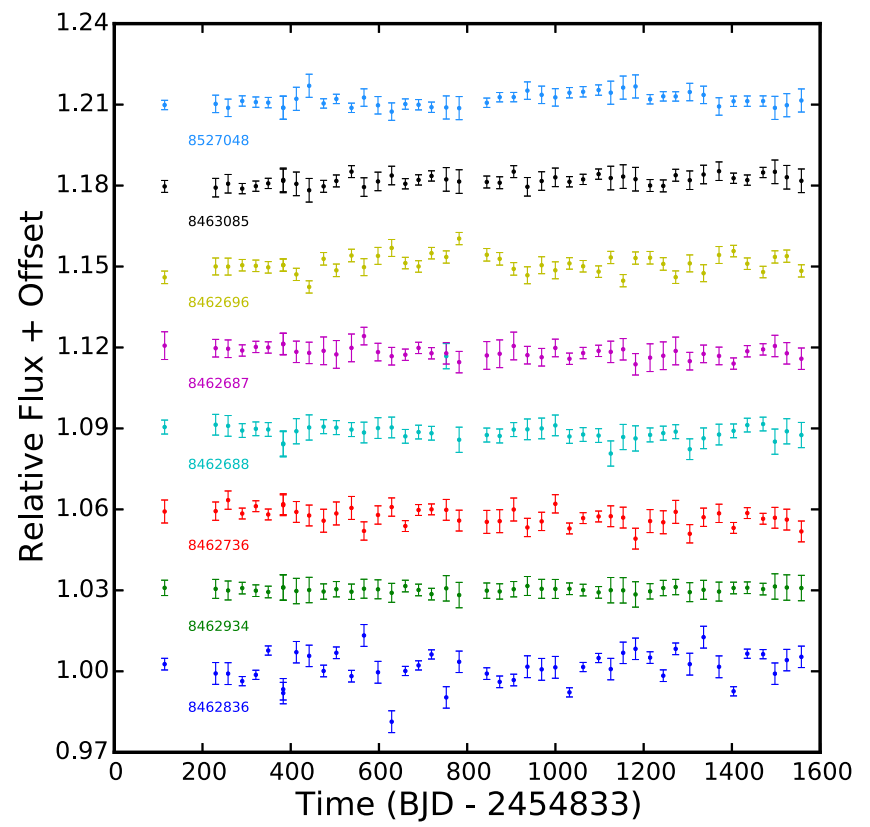

Figure 5. Photometry for eight stars produced in the same method as described in Section 2. These stars all have $11<K_{p}<14.5$ and are located within $4^{\prime}$ of KIC 8462852 on the sky. In no cases do we detect a similar dimming to that observed for KIC 8462852, suggesting that the dimming is not caused by systematics due to variability of nearby reference stars.

also be used to test the robustness of some of the signals in the PDC light curve.

\section{COMPARISON TO OTHER KEPLER STARS}

The long-term dimming of KIC 8462852 observed during the first 12 quarters of the Kepler mission differs from 0 by $8 \sigma$. In modeling the light curve, we assumed that the sources of uncertainty are entirely statistical in nature. If there are other, long-term systematics that cause a slow drift in the recorded Kepler flux from a star, those could evade our statistical analysis and cause us to erroneously record a long-term trend that does not exist. If such systematics exist, their effect should be detectable for many stars, so we can compare the observed light curve in our analysis with other stars in the Kepler field to see how often a star exhibits a dimming of $0.341 \pm 0.041 \% \mathrm{yr}^{-1}$.

\subsection{Reference Stars}

We repeat the analysis in Section 3, performing photometry on other stars that fall onto the $8 \times 8$ arcmin postcard around KIC 8462852. If long-term trends of a similar magnitude are recovered in any of these, it is an indication that the Kepler photometry is dominated by systematics from one or a few active stars in particular.

To test for such systematics, we repeat our process to develop light curves for eight other stars, all with $11<K_{p}<14.5$ and all located within $4^{\prime}$ (60 pixels) of KIC 8462852 on the sky. In these cases, KIC 8462852 was not selected by our pipeline as a potential reference star due to its photometric variability relative to the other nearby stars.

The photometry for these stars is shown in Figure 5. None of these stars exhibit a large offset in either direction around 1100 days into the mission, at the time of the rapid change in flux of KIC 8462852. We can fit a long-term trend to each of 
these stars to measure the photometric long-term variability as recorded by Kepler. In practice, we fit five parameters: a slope, a zeropoint, and three relative offsets between observing seasons as the star falls on different detectors, maximizing the likelihood of this model to the data. We also inspect each light curve by eye to look for significant deviations from nonlinearity that are similar to that detected for KIC 8462852.

For none of these stars do we recover a slope as extreme as $3.41 \pm 0.41 \mathrm{ppt} \mathrm{yr}^{-1}$. Similarly, we do not detect any $2.5 \%$ decreases in flux between days 1100 and 1250 (or at any other time), suggesting that this effect observed in the light curve of KIC 8462852 is not due to a spurious reference star, nor is there a systematic effect in all nearby stars. We do note that we recover the same changes in photometric precision as observed for KIC 8462852 as the stars move from one detector to the next while the spacecraft rolls during its orbit.

We note that for some of the targets included in Figure 5, the scatter between data points is larger than the photometric error bars. This is particularly true for KIC 8462696 and KIC 8462836. In these cases, the scatter is likely due to astrophysical variability rather than an underestimation of our uncertainties. For the former, long-cadence photometry exists from the primary mission. This star exhibits a clear photometric modulation due to starspots. Here, spots modulate the flux of the star by $3 \%$, with a rotation period of 16 days. Because the rotation period is close to half the period of FFI observations, the phase of the star's rotation when the telescope collects an FFI observation varies slowly, leading to features such as the apparent trend in the FFI flux between day 400 and 800 . We note that this time period matches well with the peak of starspot activity for this star. For KIC 8462852 we know from the Kepler data that the amplitude of photometric modulation is considerably smaller than the photometric uncertainties in the calibrated FFI data, so such a false positive trend cannot occur.

For KIC 8462836, we do not have Kepler long-cadence data. Photometry of the target is consistent with that expected of a mid-M dwarf. For mid-M dwarfs, it is not uncommon to observe photometric variability caused by starspots at the $3 \%-$ 5\% level (Basri et al. 2011), so again, the excess scatter is likely the result of starspot-induced variability that should not be present for KIC 8462852 .

\subsection{Other Nearby Stars}

We can extend our analysis to search for variations in not only the most nearby stars used as reference stars, but to all stars on the same detector. We select all stars with $K_{p}$ within 0.5 mag of KIC 8462852 and located in the same "skygroup," meaning they all fall on the same channel at the same time. We remove stars that would not be expected to provide reliable tests in a search for systematic trends in the Kepler data, including known variable stars, eclipsing binaries, and those with starspot-induced variability at the level of $1 \%$ or more through a visual inspection of the long-cadence light curves. These cuts leave 193 viable stars against which to compare.

We find that, of these 193 stars, for only 1 does our automated pipeline measure a long-term trend: KIC 8395126 appears to decrease in brightness over the Kepler mission, with a maximum likelihood measurement of the slope of

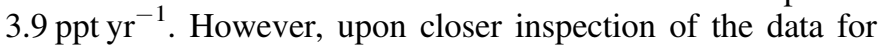
this star, we find that its center of light is approximately one pixel from the position listed in the Kepler Target Database available on MAST. Reanalyzing this target after accounting

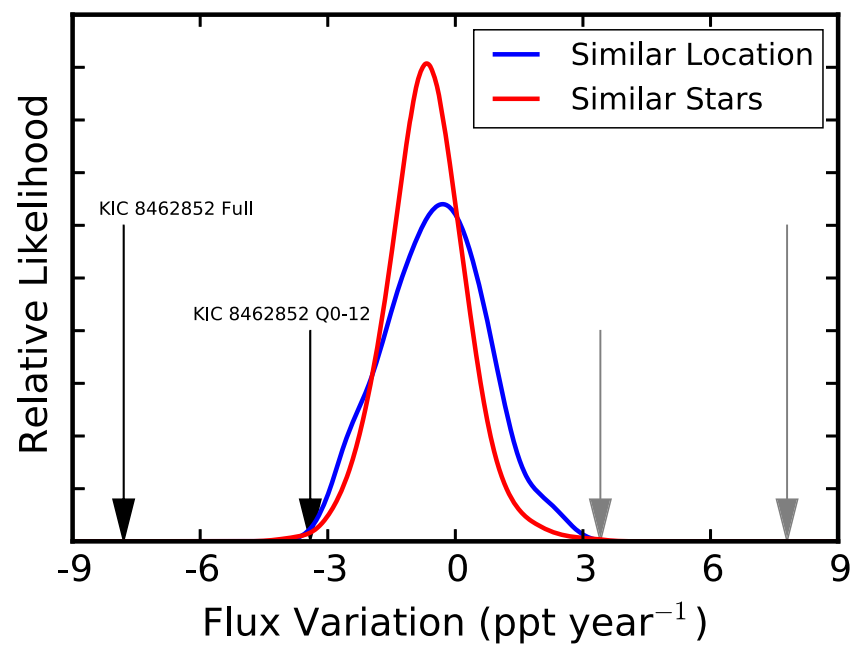

Figure 6. Kernel density estimator of the distribution of measured linear changes of the brightness of other stars observed in the Kepler FFIs. The red curve represents an analysis of other stars with similar properties to KIC 8462852; the blue represents stars of similar magnitude on the same channel of the detector. The bandwidth chosen in the creation of the KDE is equivalent to the uncertainty in the linear fit to the photometry of KIC 8462852 over the first three years of the mission. We find that $0.6 \%(0.3 \%)$ of the mass of the KDE representing stars with similar properties (similar locations) is located at variations larger than those observed in the first three years of the light curve of KIC 8462852 (represented with a black arrow). No stars in the comparison sample produce slopes as large as what would be measured by a simple linear fit to the full FFI light curve for our target (represented with a larger black arrow), nor do we observe a rapid $2.5 \%$ decrease in flux for any of these stars.

for this one-pixel offset causes the decay rate to decrease to $1.7 \mathrm{ppt} \mathrm{yr}^{-1}$, half that of KIC 8462852 . We note that repeating our analysis for KIC 8462852 while moving the centroid in any direction does not change the light curve beyond the level of the quoted photometric uncertainties. The measurements of the maximum likelihood slopes for the full sample of 193 nearby stars, again fitting for a slope and four offset terms, are distributed with a mean of $-0.16 \mathrm{ppt} \mathrm{yr}^{-1}$ and a standard

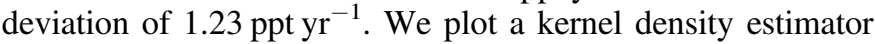
(KDE) of the distribution of fitted slopes in Figure 6. For all cases, we apply a Gaussian kernel with a bandwidth of $0.41 \mathrm{ppt} \mathrm{yr}^{-1}$ - equal to the uncertainty in the measured Q0Q12 slope for KIC 8462852 and a reasonable estimate of the typical measurement uncertainty.

We find that none of the comparison stars and only $0.3 \%$ of the mass of the KDE have a measured variation equal to or larger than the maximum likelihood value of the slope of $3.41 \mathrm{ppt} \mathrm{yr}^{-1}$ recorded over the first three years of the Kepler mission for KIC 8462852. Even more significantly, there is no mass at $7.8 \mathrm{ppt} \mathrm{yr}^{-1}$, the value recovered if one were to blindly fit a linear model to the distinctly non-linear light curve for KIC 8462852. In no cases do we observe a $2.5 \%$ dip over 6 months, as observed for KIC 8462852, suggesting that the observed behavior of our target star is unique compared to all the comparison stars on the same detector.

\subsection{Other F Stars}

We can also compare the flux variations observed for KIC 8462852 to FFI light curves for stars of similar spectral types. Here, we use the sample of KIC stars developed by Lund et al. (2016) and considered in their analysis of the DASCH plates. In that work, the authors selected 559 stars listed in the updated Kepler Input Catalog (Huber et al. 2014) with inferred stellar 
effective temperatures within $100 \mathrm{~K}$, radii within $5 \%$, and $\log g$ within $10 \%$ of KIC 8462852 .

From this sample, we remove all stars for which we do not expect to be able to acquire reliable photometry or observe long-term photometric trends over four-year baselines. We remove stars with brighter neighbors located within 10 pixels of the target star so that their flux is likely to leak into our own aperture. We also remove stars near the edge of the detector and stars that do not fall onto the Kepler detectors in at least three of the four observing seasons. We remove stars that saturate the detector, and also those that are intrinsically variable, including contact binaries, stars that vary due to spots at larger than the $2 \%$ level, and pulsating variable stars. We note that the two targets that Lund et al. (2016) noted as longterm variable, KIC 3868420 and KIC 11802860, both are removed by this last requirement. The former exhibits $15 \%$ variability with a five hour period in the Kepler light curve; Nemec et al. (2013) suggest it may be a high-amplitude Delta Scuti. The latter exhibits $40 \%$ variability with a $16.5 \mathrm{hr}$ period in its Kepler light curve; the star is also known as AW Dra and has long been known as an RR Lyrae (Castellani et al. 1998).

The long-term variability observed by Lund et al. may be the result of unfortunate timing of the observations across different phases of the star's light curve, causing an apparent long-term trend. This is plausible, as the claimed photometric trends have different signs in the pre- and post-1970 subsets of the DASCH data. The long-term variability could also be a case of rapid stellar evolution: Stellingwerf (2013) suggest that RR Lyrae stars may be undergoing rapid mass-loss, which may lead to a dimming over a timescale of decades.

We note that the overall distribution of $\mathrm{F}$ star variability is

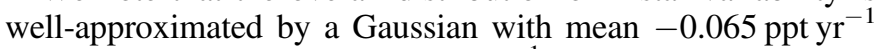

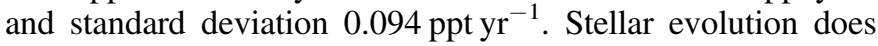
not explain a long-term trend in the typical F star; detection of a nonzero mean dimming is likely indicative of a systematic in the Kepler data that is not understood. We repeat our analysis on these stars. Again, none of the target stars exhibit a 3\% change in flux over the duration of the Kepler mission, nor do any stars change in flux by $2.5 \%$ over six months. Of all stars analyzed in this work, the rapid dimming between quarters 12 and 15 appears to be unique to KIC 8462852 . In fact, only a single other star, KIC 5868753, has an observed change in brightness larger than the maximum likelihood slope for KIC 8462852 from the first three years of the Kepler mission.

\section{DISCUSSION}

The Kepler FFI light curve for KIC 8462852 features a longterm slow dimming over the first three years of the mission. Formally, the observed slope is statistically significant, but from our analysis of nearby stars it is likely that systematic uncertainties dominate over the statistical uncertainties. There are no stars that we detect that exhibit a $7.8 \mathrm{ppt} \mathrm{yr}^{-1}$ dimming, the level that a simple linear model would produce when fit to the full, non-linear data set. Additionally, there are no stars that exhibit a $2.5 \%$ decrement in flux over approximately six months.

Even if we consider only the relatively modest dimming over the first three years of the Kepler mission, there is only a single star that exhibits a slope that is at least as large in magnitude as the one observed here.

The observed behavior of the star is thus very likely astrophysical in nature, suggesting this star is indeed undergoing some process leading to a decrease in its observed brightness over the span of the Kepler mission. In the remainder of this section we consider possible interpretations of this result.

\subsection{Comparison to DASCH Photometry}

As mentioned in Section 1, Schaefer (2016) used 99 years of photometry from the DASCH project to analyze the behavior of KIC 8462852, finding a decrease in brightness of $14 \%$ from 1890 to 1989 . Hippke et al. (2016) performed an independent analysis of the DASCH photometry, confirming that the photographic data yield a fainter magnitude for KIC 8462852 in the late 20th century compared to the end of the 19th century. However, Hippke et al. argue that the DASCH measurements from 1890 to 1952 are best described by a constant brightness and measurements from 1967 to 1989 are best described by a different (fainter) constant brightness, with systematic errors accounting for the offset. We note that such systematic offsets have not been detected in DASCH light curves by the DASCH team (Laycock et al. 2010; Tang et al. 2013a, 2013b).

The unfortunate gap of 15 years with very few observations in the middle of the century makes it difficult to distinguish between a real astrophysical variation and a systematic offset. Which explanation is more preferable then depends on one's assessment of which scenario is more likely (or less unlikely): a star steadily fading for a century or a change in the photometric calibration of the Harvard plates around 1962. The fading that we detect from 2009 to 2013 with the Kepler FFI images does not necessarily represent a confirmation that KIC 8462852 also dimmed over the preceding 129 years, but could make that interpretation of the DASCH data more plausible.

Both previous analyses considered only linear models for the flux of the star as a function of time, as the DASCH data are insufficient for constraining more complex models. The Kepler FFI data are plainly non-linear, suggesting that the true light curve during the 20th century is likely more complex than either simple model. Additionally, a decrease of $3.5 \%$ in the flux of KIC 8462852 is four times what would be expected over the four-year Kepler mission from Schaefer's century-long light curve. Conversely, if the brightness change over the duration of the DASCH survey were as extreme as that observed during the Kepler mission, we would expect the star to have decreased in brightness by $60 \%$ over the 20th century, which does not appear to be consistent with comparisons of archival and modern (e.g., Boyajian et al. 2016) measurements. As there is a gap of two decades between the end of the DASCH data and the start of the Kepler mission, a direct comparison between the two is difficult. Still, it is clear that neither a linear decrease in flux nor a constant flux model provide a good description of our observations, so there is no reason to expect the behavior at earlier times to have followed a simpler form.

\subsection{Instrumental Effects}

The observed dimming does not seem to be associated with any instrumental effect. Some known effects could cause a $2.5 \%$ decrement in the observed flux from a star. The most common such effect is a sudden pixel sensitivity dropout (SPSD), in which a pixel abruptly decreases in sensitivity, leading to a decrease in the observed flux on that pixel. The 
pixel generally recovers in sensitivity over a few hours, but does not return to the same sensitivity level as before (Smith et al. 2012). An SPSD could not explain our observed light curve, as the observed flux decrement is visible in all four detector orientations, so the same SPSD would have had to occur on all four apertures, at the same level, at approximately the same time.

Similarly, we do not expect this effect to be the result of any calibration issues during the processing of the FFIs. The calibration pipeline accounts for the bias and dark current of the detector, cosmic rays, smearing effects from reading out without a shutter, and distortions induced from the readout electronics (Quintana et al. 2010). Most of these effects are dependent on the location of the star, rather than the physical properties of the star itself, so we would not expect them to induce a dimming observed over all four modules. The exception is the smear correction, but the smear is wellunderstood and can be robustly estimated from the data. Significant errors in the smear correction would be easily observable in the FFI data itself as "trails" in the FFI image near the star, which are not detected in these observations. Therefore, we can rule out instrumental effects or calibration errors as a plausible source of the observed variability.

\subsection{Comparison with Long-cadence Light Curve}

The long-cadence light curves produced by the Kepler mission are accurate over timescales of hours to days. Over longer timescales, systematics overwhelm long-term astrophysical photometric variations. This is largely because of two effects. First, there is a lack of reference stars, as only $\approx 2 \%$ of all stars in the Kepler field are targeted for photometry at a 30minute cadence. Therefore, nearby stars that should share systematics are often not observed at the cadence that would be necessary for co-trending to identify shared systematics. Second, the apertures downloaded by Kepler are typically smaller than the PSF of each individual star (Figure 2), so that small changes in the pointing of the telescope can cause significant flux variations, overwhelming any signal from the star itself. With the FFI data in hand, we create our own longcadence light curve from the pixel-level data.

As the FFI data provide absolute calibration of the Kepler data once per month, the FFI data can be combined with the flattened Kepler long-cadence light curve to show one plausible iteration of the true astrophysical long-cadence light curve of KIC 8462852, with instrumental effects removed. To create this light curve, we fit a cubic spline to the FFI data using the spline tool in scipy.optimize. We use the measured photometric precision for each FFI as the input weights, applying a smoothing factor of 50. The resultant smooth light curve is then multiplied by the flattened light curve of Section 3.3 to create a realistic long-cadence light curve that captures both the short-term variability and the long-term dimming observed during the Kepler mission. These data are plotted in Figure 3 and included in the same "data behind the figure" as both the intermediate, flattened light curve described in the previous paragraph and the smooth spline fit to the FFI data. As the data are not calibrated between the FFIs, and each FFI observation has an associated photometric uncertainty, this long-cadence light curve represents one possible realization of the photometric variability of KIC 8462852 and should only be considered in that context.

\subsection{Background Contamination}

Approximately 25 arcsec from KIC 8462852 is KIC 8462860, a star 3.65 mag fainter on the edge of our photometric aperture. As approximately half of the star falls in our simple aperture, we would expect it to contribute approximately $1.8 \%$ of the total flux in our aperture. This star cannot explain the long-term trend over the first three years of the mission unless it were to decrease in flux by $50 \%$; it cannot explain the rapid dimming event unless it disappeared entirely (which is obviously not the case).

KIC 8462860 could explain the observed rapid dimming if the star had a large proper motion and moved out of the aperture during Quarters 13 and 14. However, in the PPMXL catalog (Roeser et al. 2010) its proper motion is $\mu_{\text {R.A. }}=$ $2.4 \pm 4.1 \mathrm{mas} \mathrm{yr}^{-1}, \quad \mu_{\text {decl. }}=0.3 \pm 4.1 \mathrm{mas} \mathrm{yr}^{-1}$. The first Gaia data release does not include a proper motion measurement of KIC 8462860. By combining the Gaia measurement of the position of the star (Lindegren et al. 2016) with the position recorded in the 2MASS point source catalog (Cutri et al. 2003),

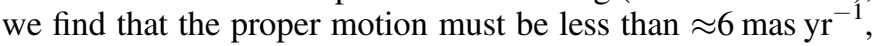
in line with the PPMXL result. The star is listed in the KIC as having a temperature of $5464 \mathrm{~K}$, and its magnitude and colors $\left(m_{r}=15.6, r-J=1.4, J-K=0.5\right)$ are broadly consistent with a late $\mathrm{G}$ or early $\mathrm{K}$ dwarf at several hundred parsecs or an evolved $\mathrm{G} / \mathrm{K}$ star at larger distances. The photometry is not consistent with what would be expected of a nearby star.

If the star did have a large proper motion, we would see it move by several pixels over the course of the mission, which visual inspection of the FFI data does not show. Moreover, to move far enough so that the entire core of the PSF moved across the edge of our aperture over a span of approximately six months, the proper motion would need to be approaching $10 \operatorname{arcsec}_{\mathrm{yr}^{-1}}$. In this case, we would expect the star to traverse the entire aperture over the four years of FFI data, which we do not observe.

Perhaps most significantly, the same light curve as shown in Figure 3 is recovered when we mask the pixels corresponding to KIC 8462860 , or if we modify the size of our aperture to either move this other star fully inside or outside of the aperture rather than on the edge. Therefore, we can exclude the possibility that the observed variations in the light curve are caused by the presence of KIC 8462860 . Moreover, we do not detect the presence of any centroid shifts correlated with the measured flux in the light curve of this star, suggesting this result is not the effect of the neighboring star or changes in the underlying flat-field. We can confidently rule out background contamination as an explanation of our light curve .

\subsection{Transiting Material}

We note that the shape of the light curve after Quarter 12 appears broadly similar to that of a transit event. Over six months, the rapid dimming could represent the ingress of material blocking the stellar disk, leading to a decrease in observed flux. The primary mission then ends before third contact is observed. Clouds of transiting material due to disintegrating bodies have been observed in Kepler data, but only with very short orbital periods (Rappaport et al. 2014; Sanchis-Ojeda et al. 2015). In the case of KIC 8462852, there are problems with this model. First, it does not explain the observed long-term dimming over the first three years of the Kepler mission, nor does it explain the long-term trend 
observed over the past 100 years through the DASCH data. A single transit model also does not explain the phenomena observed by Boyajian et al. (2016), especially those dips before the time of ingress. Nevertheless, we investigate whether it is a possible explanation for the rapid dimming.

A transit event with a timescale similar to that observed here is not unprecedented. $\epsilon$ Aurigae is transited every 27 years, with a transit lasting approximately 1.5 years (Kopal 1954; Huang 1965). Recently, Rodriguez et al. (2016) observed transits of TYC 2505-672-1 with a period of 69.1 years and a duration of 3.45 years. In both cases, the primary is orbited by a hot source that hosts an extended disk of circumstellar material. Kloppenborg et al. (2010) confirmed this model with closurephase interferometric imaging, directly imaging the disk occulting $\epsilon$ Aurigae.

For both $\epsilon$ Aurigae and TYC 2505-672-1, the disk is optically thick and circumstellar around a binary companion. The submillimeter observations of Thompson et al. (2016) place a limit of $7.7 M_{\oplus}$ of material within 200 au of KIC 8462852 , ruling out a direct analog to these other systems.

First, we consider that this signal represents the transit of a solid body across the stellar disk of KIC 8462852. In this scenario the observed timescales of the event constrain the size and distance of the eclipsing object. For an optically thick transiting object, the $2.5 \%$ transit depth indicates a minimum radius of $0.15 R_{*}$ (Boyajian et al. 2016 estimate a radius of $1.58 R_{\odot}$ for KIC 8462852 ). If the transiting body is in a Keplerian orbit, the extremely slow ingress time and long transit duration place it at the implausibly large distance of $\sim 10 \mathrm{pc}$, with a transit probability of $\sim 10^{-9}$. Note that while a six-month transit ingress and transit duration of more than six months are similar to $\epsilon$ Aurigae and TYC 2505-672-1, this case is distinct from those because of the much smaller radius of KIC 8462852. The transit of a main sequence star is necessarily much shorter than that of a supergiant with $R \gtrsim 50 R_{\odot}$. Even if every star had a companion at $10 \mathrm{pc}$, then given $10^{4}$ Kepler missions we would expect to observe one such transit event; we can confidently disfavor this hypothesis.

If the transiting material is instead an optically thin cloud, then its size could be comparable to or larger than that of KIC 8462852. The optical depth of such a cloud would be $\tau \approx 0.025$. Because the orbiting body considered in the previous paragraph was already a significant fraction of the stellar radius, though, a much bigger cloud does not qualitatively change the result: in order for the transit ingress to last $\sim 180$ days, a cloud in a Keplerian orbit would need to be located $\gtrsim 1 \mathrm{pc}$ away from the star. We thus conclude that a simple transit of any kind of orbiting object is not a reasonable explanation for the rapid fading that begins in Quarter 12. However, this conclusion relies on the assumption that the duration of the transit ingress is set by the orbital velocity of the transiting body.

More complex scenarios in which the ingress timescale reflects the spreading of debris along its orbit after a recent collision or the precession of an occulting disk into our line of sight could perhaps explain the appearance of a transit. For example, a cloud that slowly increases in density would manifest itself in the light curve as inducing a change in flux similar to that observed in Quarter 12. However, to produce the apparent flat bottom of the supposed transit event, such a cloud would then need to be extended over a fraction of its Keplerian orbit and would need to maintain an approximately constant density through its entire length as it passes in front of this star.

As illustrated above, it is very difficult to come up with a physical model that can even qualitatively explain all of the major features of KIC 8462852's photometric behavior simultaneously. Of the ideas that have been proposed so far, we suggest that the most promising explanation involves a recent collision between large bodies (planetesimals or comets) in the KIC 8462852 system, leading to a spreading of debris as in the previous paragraph. In this picture, a recent collision could create a cloud of circumstellar material and push a family of objects into a highly eccentric orbit, analogous to the period of late heavy bombardment observed in our own solar system. However, this idea does not naturally account for the steady decline in the flux of KIC 8462852 in the years preceding the more rapid dimming and the concentrated sequence of dips. The data presented in this paper cannot fully exclude any of these models, but we note that the circumstellar dust and debris produced by such an event are unlikely to maintain this arrangement for long timescales. The submillimeter limits of Thompson et al. (2016) and continued photometric monitoring will significantly constrain future models that attempt to invoke circumstellar dust or transiting models to explain this light curve. Transiting material remains as a plausible explanation for the KIC 8462852 light curve, but requires particular, a priori unlikely density profiles for the circumstellar material in order to match the data.

\subsection{A Polar Spot}

Under certain conditions, a long-lasting spot growing at polar latitudes on the surface of KIC 8462852 could possibly reproduce the long-term light curve observed here. While polar spots have not previously been detected on an F3V dwarf star, they have been observed on the surface of an F9V star, albeit one in a tight (1.15 day) binary with another stellar companion (Strassmeier \& Rice 2003). Through interferometric aperture synthesis imaging, polar spots on more evolved stars have been seen to evolve on similar timescales to the flux variability observed here (Roettenbacher et al. 2016).

For a polar spot to create the observed decrease in flux in the light curve, the projection of the polar region onto the observer's line of sight would need to be large enough to allow for a large starspot to be observed. At high (edge-on) inclinations, a polar spot would be foreshortened, diminishing its effect on the light curve. Moreover, limb darkening would decrease the overall contribution of the polar regions on the light curve. At lower (more face-on) inclinations, the pole would always be visible and a polar spot would have a larger effect on the overall light curve. Boyajian et al. (2016) measure a rotation period and $v \sin i$ of the star and combine these with an estimate of the radius to infer an inclination of $68^{\circ} \pm 29^{\circ}$ at $68 \%$ confidence, leaving open the possibility that the pole can indeed be observed at all times well away from the edge of the stellar disk.

If a spot were growing near the polar latitudes of the surface of the star between Quarters 13 and 15, we might expect a corresponding increase in overall magnetic activity, leading to an increase in starspots at other latitudes over this time period. This indeed appears to be the case. Boyajian et al. (2016) measure a periodic signal with a period of 0.88 days, as well as additional signals at 0.90 and 0.96 days, all of which change in intensity during the Kepler mission. The authors suggest that 
this signal may be induced by starspots. Spots at different latitudes evolving and rotating differentially would produce a signal like this one. The strength of the signal they observe grows between days 1100 and 1300 of the mission, suggesting either an increase in the number of spots or an increase in their coherence. Interestingly, the growth of the signal corresponds to the time of rapid decrease in total flux recorded in the FFI images.

The inclination of KIC 8462852 and the coincidence between the spot activity and the rapid dimming do not present a confirmation of a polar spot. A long-lasting polar spot itself would be remarkable given the F3V spectral type of the star, but with the current data the hypothesis cannot be excluded. Broadly, the FFI observations are consistent with the growth of a polar spot that grows at a similar time to the growth of spots at lower latitudes. Spots, however, cannot explain the shortterm dips originally observed by Boyajian et al. (2016). More observations are needed to separate the spot hypothesis from other possible explanations of the observed light curve. As the polar spot hypothesis cannot account for the short-term dips of Boyajian et al. (2016), it does not seem to be a particularly likely scenario, but cannot be ruled out given the available data.

\subsection{Additional Observations}

In this work, we do not present a model that can explain the entire suite of observations of KIC 8462852. There are now three distinct photometric variations observed: rapid, irregular decreases of $10 \%$ or more in flux lasting for a few days, a $2.5 \%$ decrease in flux lasting for at least one year, and a likely longterm dimming perhaps spanning more than a century. Additional observations would be helpful in order to better understand physical phenomena that could cause any or all of these events.

Multi-color photometry is essential to help characterize this star. If any of these events are caused by solid bodies, we would expect the photometric variations to be largely achromatic. However, if they are caused by a cloud of dust and gas, we would expect the cloud to redden the star. Similarly, spots with a lower effective temperature than the rest of the stellar surface would cause an apparent change in the color of the star. as they cover more of the stellar disk. A transiting cloud would be expected to induce dips that are largely periodic, while changes in the spot patterns would not necessarily be periodic. High-precision observations of the color of KIC 8462852 over time could explain the nature of each of these events, supporting or ruling out the transiting cloud or spot hypotheses. Similarly, additional IR and submillimeter observations of the system could be used to place tighter upper limits on the amount of circumstellar material surrounding the star.

Additional RV monitoring to search for companions in fewau orbits around the star, especially those that could hold together a disk of circumstellar material into a gravitationally bound system, would also be useful. Boyajian et al. (2016) obtained four high-resolution spectra over the course of approximately 500 days. The measured RV of KIC 8462852 in these observations has a scatter of $0.3 \mathrm{~km} \mathrm{~s}^{-1}$ and each observation has a precision of $0.4 \mathrm{~km} \mathrm{~s}^{-1}$. While the data are consistent with no RV variations, continued monitoring could detect the presence of massive companions on wider orbits. High-resolution spectra could also be used to probe any evolution in the magnetic activity of the star correlated with the growth or decay of spots.

\section{CONCLUSIONS}

Recently, multiple analyses of DASCH photometry have produced conflicting results about the possible detection of a long-term dimming of KIC 8462852 by $0.165 \pm 0.013 \mathrm{mag}$ over the 20th century, or $0.152 \pm 0.012 \% \mathrm{yr}^{-1}$. The dimming of a star at that rate should be detectable in Kepler data. Here, we analyze monthly Kepler FFI images to search for similar dimming in the FFI light curve of KIC 8462852.

We perform aperture photometry on KIC 8462852 and seven nearby comparison stars. We observe that during the first three years of the Kepler mission, the star dimmed at a rate of $0.341 \pm 0.041 \% \mathrm{yr}^{-1}$. Over the following six months, the star decreased in brightness by $2.5 \%$, then remained at that level for the duration of the primary Kepler mission. This result is not sensitive to the size of the chosen aperture or the particular choice of reference stars.

We then compare this result to a similar analysis of other stars of similar brightness on the same detector, as well as stars with similar stellar properties, as listed in the KIC, in the Kepler field. We find that $0.3 \%$ of stars on the same detector and $0.6 \%$ of stars with similar stellar properties exhibit a longterm trend consistent with that observed for KIC 8462852 during the first three years of the Kepler mission. However, in no cases do we observe a flux decrement as extreme as the $2.5 \%$ dip observed in Quarters 12-14 of the mission. The total brightness change of KIC 8462852 is also larger than that of any other star we have identified in the Kepler images.

Broadly speaking, the morphology of the light curve is generally consistent with the transit of a cloud of optically thick material orbiting the star. Such a dust cloud could be small enough to evade submillimeter detection in the analysis of Thompson et al. (2016), who place a limit of $7.7 M_{\oplus}$ of material orbiting KIC 8462852 . The break-up of a small body or a recent collision that could produce a cloud of material could also plausibly produce a family of comets that transit the host star together as one group (Bodman \& Quillen 2016), explaining the light curve observed by Boyajian et al. (2016). However, in order to match the observed time of ingress and transit duration, some fine-tuning is required. To explain the transit ingress timescale, the cloud would need to be at impossibly large distances from the star or be slowly increasing in surface density. The flat bottom of the transit would then suggest a rapid transition into a region of uniform density in the cloud, which then continues to transit the star for at least the next year of the Kepler mission. Moreover, such a model does not naturally account for the long-term dimming in the light curve observed in both DASCH and the Kepler FFI data, suggesting that this idea is, at best, incomplete.

There is no known or proposed stellar phenomenon that can fully explain all aspects of the observed light curve. Non-stellar explanations, such as circumstellar material, offer the best opportunity to provide an explanation for the observed light curve, but simple models are unable to match the light curve as observed over the last century. We strongly encourage further refinements, alternative hypotheses, and new data in order to explain the full suite of observations of this very mysterious object. 
We thank Jason Dittmann and Jieun Choi (Harvard) for conversations about data analysis and figure design that improved the quality of this paper. We thank John Brewer (Yale/Columbia) for finding a typo in Equation (1) in an earlier version of this paper. We also thank Rachael Roettenbacher (Stockholm University) and John Johnson (Harvard) for discussions of starspots, Ryan Foley (UC Santa Cruz) for initial conversations about photometry and the DASCH results, George Preston (Carnegie) for insights into variable stars, and Eugene Chiang (UC Berkeley) and Jason Wright (Penn State) for ideas about other potentially similar systems.

B.T.M. is supported by the National Science Foundation Graduate Research Fellowship under grant No. DGE-1144469.

Funding for Kepler, the 10th Discovery mission, was provided by NASA's Science Mission Directorate. We are grateful to the entire Kepler team, past and present. Their tireless efforts were essential to the tremendous success of the mission and the successes of $K 2$, present and future.

All of the data presented in this paper were obtained from the Mikulski Archive for Space Telescopes (MAST). STScI is operated by the Association of Universities for Research in Astronomy, Inc., under NASA contract NAS5-26555. Support for MAST for non-HST data is provided by the NASA Office of Space Science via grant NNX09AF08G and by other grants and contracts.

This research has made use of the SIMBAD database, operated at CDS, Strasbourg, France and NASA's Astrophysics Data System.

Facility: Kepler.

\section{REFERENCES}

Abeysekara, A. U., Archambault, S., Archer, A., et al. 2016, ApJL, 818, L33

Barnes, S. A., Weingrill, J., Fritzewski, D., Strassmeier, K. G., \& Platais, I. 2016, ApJ, 823, 16

Basri, G., Walkowicz, L. M., Batalha, N., et al. 2011, AJ, 141, 20

Bastien, F. A., Stassun, K. G., Basri, G., \& Pepper, J. 2013, Natur, 500, 427

Bedding, T. R., Mosser, B., Huber, D., et al. 2011, Natur, 471, 608

Bodman, E. H. L., \& Quillen, A. 2016, ApJL, 819, L34

Borucki, W. J., Koch, D., Basri, G., et al. 2010, Sci, 327, 977

Boyajian, T. S., LaCourse, D. M., Rappaport, S. A., et al. 2016, MNRAS, 457, 3988

Boyajian, T. S., von Braun, K., van Belle, G., et al. 2012, ApJ, 757, 112

Bryson, S. T., Tenenbaum, P., Jenkins, J. M., et al. 2010, ApJL, 713, L97

Butler, R. P., Marcy, G. W., Williams, E., et al. 1996, PASP, 108, 500

Caldwell, D. A., Kolodziejczak, J. J., Van Cleve, J. E., et al. 2010, ApJL, 713, L92

Carter, J. A., Agol, E., Chaplin, W. J., et al. 2012, Sci, 337, 556

Castellani, V., di Paolantonio, A., Piersimoni, A. M., \& Ripepi, V. 1998, A\&A, 333,918

Chaplin, W. J., Basu, S., Huber, D., et al. 2014, ApJS, 210, 1
Cutri, R. M., Skrutskie, M. F., van Dyk, S., et al. 2003, 2MASS All Sky Catalog of Point Sources, http://irsa.ipac.caltech.edu/applications/Gator/ Finkbeiner, D. P., Schlafly, E. F., Schlegel, D. J., et al. 2016, ApJ, 822, 66 García, R. A., Hekker, S., Stello, D., et al. 2011, MNRAS, 414, L6

Gilliland, R. L., Chaplin, W. J., Dunham, E. W., et al. 2011, ApJS, 197, 6

Grindlay, J., Tang, S., Simcoe, R., et al. 2009, in ASP Conf. Ser. 410, Preserving Astronomy's Photographic Legacy: Current State and the Future of North American Astronomical Plates, ed. W. Osborn \& L. Robbins (San Francisco, CA: ASP), 101

Haas, M. R., Batalha, N. M., Bryson, S. T., et al. 2010, ApJL, 713, L115

Harp, G. R., Richards, J., Shostak, S., et al. 2016, ApJ, 825, 155

Hippke, M., Angerhausen, D., Lund, M. B., Pepper, J., \& Stassun, K. G. 2016, ApJ, 825, 73

Huang, S.-S. 1965, ApJ, 141, 976

Huber, D., Silva Aguirre, V., Matthews, J. M., et al. 2014, ApJS, 211, 2

Jenkins, J. M., Caldwell, D. A., Chandrasekaran, H., et al. 2010, ApJL, 713, L87

Kinemuchi, K. 2011, in RR Lyrae Stars, Metal-Poor Stars, and the Galaxy, Vol. 5, ed. A. McWilliam (Pasadena, CA: The Observatories of the Carnegie Institution of Washington), 74

Kloppenborg, B., Stencel, R., Monnier, J. D., et al. 2010, Natur, 464, 870

Kopal, Z. 1954, Obs, 74, 14

Laycock, S., Tang, S., Grindlay, J., et al. 2010, AJ, 140, 1062

Lindegren, L., Lammers, U., Bastian, U., et al. 2016, A\&A, in press (arXiv:1609.04303)

Lisse, C. M., Sitko, M. L., \& Marengo, M. 2015, ApJL, 815, L27

Lund, M. B., Pepper, J., Stassun, K. G., Hippke, M., \& Angerhausen, D. 2016, ApJ, submitted (arXiv:1605.02760)

Magnier, E. A., Schlafly, E., Finkbeiner, D., et al. 2013, ApJS, 205, 20

Marengo, M., Hulsebus, A., \& Willis, S. 2015, ApJL, 814, L15

McQuillan, A., Mazeh, T., \& Aigrain, S. 2014, ApJS, 211, 24

Meibom, S., Barnes, S. A., Platais, I., et al. 2015, Natur, 517, 589

Miglio, A., Brogaard, K., Stello, D., et al. 2012, MNRAS, 419, 2077

Molnár, L., Pál, A., Plachy, E., et al. 2015, ApJ, 812, 2

Mosser, B., Goupil, M. J., Belkacem, K., et al. 2012, A\&A, 548, A10

Nemec, J. M., Cohen, J. G., Ripepi, V., et al. 2013, ApJ, 773, 181

Quintana, E. V., Jenkins, J. M., Clarke, B. D., et al. 2010, Proc. SPIE, 7740, $77401 X$

Rappaport, S., Barclay, T., DeVore, J., et al. 2014, ApJ, 784, 40

Rappaport, S., Levine, A., Chiang, E., et al. 2012, ApJ, 752, 1

Rodriguez, J. E., Stassun, K. G., Lund, M. B., et al. 2016, AJ, 151, 123

Roeser, S., Demleitner, M., \& Schilbach, E. 2010, AJ, 139, 2440

Roettenbacher, R. M., Monnier, J. D., Korhonen, H., et al. 2016, Natur, 533,217

Sanchis-Ojeda, R., Rappaport, S., Pallè, E., et al. 2015, ApJ, 812, 112

Schaefer, B. E. 2016, ApJL, 822, L34

Schuetz, M., Vakoch, D. A., Shostak, S., \& Richards, J. 2016, ApJL, 825, L5

Silva Aguirre, V., Davies, G. R., Basu, S., et al. 2015, MNRAS, 452, 2127

Smith, J. C., Stumpe, M. C., Van Cleve, J. E., et al. 2012, PASP, 124, 1000

Stellingwerf, R. F. 2013, arXiv:1310.0535

Strassmeier, K. G., \& Rice, J. B. 2003, A\&A, 399, 315

Szabó, R., Kolláth, Z., Molnár, L., et al. 2010, MNRAS, 409, 1244

Tang, S., Grindlay, J., Los, E., \& Servillat, M. 2013a, PASP, 125, 857

Tang, S., Sasselov, D., Grindlay, J., Los, E., \& Servillat, M. 2013b, PASP, 125,793

Thompson, M. A., Scicluna, P., Kemper, F., et al. 2016, MNRAS, 458, L39 van Saders, J. L., Ceillier, T., Metcalfe, T. S., et al. 2016, Natur, 529, 181

Wright, J. T., Cartier, K. M. S., Zhao, M., Jontof-Hutter, D., \& Ford, E. B. 2016, ApJ, 816, 17 